second course of antibiotics twice as frequently? And what if $C$ difficile is more common with antibiotic A, with a measurable incidence of prolonged hospital stay as a consequence? The total cost of using the superficially cheaper product may actually surpass that of the more expensive agent.

It is possible to construct complex models setting out all the possible ramifications of a clinical decision, and to attach a cost to these options. It is, however, time-consuming to build up models from scratch for every possible recommendation, and it is acceptable to focus only on particular issues. For example, there is little point in performing complex analysis where one option clearly gives better clinical outcomes than the alternatives, unless the superior option is particularly expensive. The definition of "expensive" is, of course, debatable, and one reason many feel uncomfortable with health economics is that it forces decisions about the amount one should be prepared to pay for clinical benefit. Quite properly, the decision about such thresholds is not the province of the guideline group themselves.

Detailed health economic input is relatively new to many guidelines, and there are issues around the integration of this with the purely clinical work which are not fully resolved. ${ }^{3}$ However, it will be increasingly difficult to produce acceptable guidelines without some consideration of economic consequences.

\section{DISSEMINATION AND IMPLEMENTATION}

Although there is a substantial literature about implementation of guidelines, ${ }^{4}$ it is still not clear how best to ensure that this happens. Producing clearly written material, in different versions for different audiences, is important but not sufficient by itself. Various methods have been tried to enhance take-up of guidance using both carrot and stick, but there does not seem to be a generalisable consistent means of achieving success. Overall, the best results have been obtained where it has been possible to empower patients to promote guideline adherence, but this is difficult to organise except in very simple situations such as encouraging influenza vaccination.

The BTS has shown that dissemination based around genuinely helpful materials can work well. The COPD Consortium, founded to promulgate the 1997 COPD guideline, was hugely successful. The dissemination efforts which followed the BTS/SIGN asthma guideline launch in $2003^{5}$ stimulated over 200000 downloads in the first 18 weeks, and larger numbers still if the associated teaching materials are taken into account.

\section{CONCLUSION}

It is harder to produce methodologically acceptable guidelines than it was 10-15 years ago, but important that technical validity is not achieved at the expense of clinical usefulness. Although there are now national and international organisations involved in guideline production, I believe it is important that specialist societies continue to be involved. They can best judge where guidance is necessary on the "smaller" topics; for guidance on the big diseases, where those outside the specialty have a significant stake and where the amount of evidence may stretch resources, the future may lie in collaboration with others, but our input is still crucial. The BTS can look back with pride on its previous work; it is also well placed to move forward as guideline production evolves.

Thorax 2007;62:1022-1024.

doi: 10.1136/thx.2007.090944

Correspondence to: Dr Bernard G Higgins, Freeman Hospital, Newcastle upon Tyne NE7 7DN, UK; b.g.higgins@nd.ac.uk

Competing interests: None.

\section{REFERENCES}

1 British Thoracic Society/Scottish Intercollegiate Guideline Network. British guideline on the management of asthma. Thorax 2003;58(Suppl I): i1-94.

2 The GRADE Working Group. Grading quality of evidence and strength of recommendations. BMJ 2004;328: 1490-4

3 Scottish Intercollegiate Guideline Network. SIGN 50: a guideline developers' handbook. www.sign.ac.uk/guidelines/fulltext/50/section6 (accessed 20 September 2007).

4 Grimshaw JM, Thomas RE, MacLennan G, et al. Effectiveness and efficiency of guideline dissemination and implementation strategies. Health Technol Assess 2004;8:iii-iv:1-72.

5 Dennis SM, Edwards S, Partridge MR, et al. The dissemination of the British guideline on the management of asthma 2003. Respir Med 2004;98:832-7.

\title{
Hypercapnic respiratory failure: from the past to the future
}

\section{John Shneerson}

\section{The importance of non-invasive ventilation}

I t seems surprising now, but the concept of respiratory failure is quite a recent development in respiratory medicine. Up until the middle of the 20th century, when chest physicians were dealing primarily with tuberculosis, it was virtually overlooked. ${ }^{1}$ The principles of the biochemical control of respiration had only recently been demonstrated, arterial blood analysis was difficult and timeconsuming, and the understanding of acid-base metabolism was primitive by modern standards. The work of Moran Campbell and his colleagues, ${ }^{2}$ however, clarified the physiological basis of the clinical presentations of respiratory failure. The "blue bloater" who hypoventilated and developed right heart failure was contrasted with the "pink puffer" who suffered breathlessness but maintained a normal carbon dioxide tension and near normal oxygen tension. The adverse effect of supplemental oxygen of removing the hypoxic drive and exacerbating respiratory failure was recognised and controlled oxygen therapy using Venturi masks became established.

At the same time as these ideas about respiratory failure due to chronic obstructive pulmonary disease (COPD) were developing, a separate line of thought was evolving. Poliomyelitis-which until the early 1900s had caused only sporadic infections-began to appear in epidemics in the USA and many northern European countries and left thousands of children and some adults paralysed. These epidemics reached their peak in the 1950s until the advent of the Salk and Sabin immunisations. Acute hypercapnic respiratory failure due to respiratory muscle weakness was often fatal.

The first effective treatment was the tank ventilator or iron lung which was developed in 1928 by Drinker, an engineer with the Consolidated Gas Company of New York City. ${ }^{3}$ This comprised a negative pressure chamber which surrounded the body up to the neck and which was connected to a powerful pump 
capable of evacuating the chamber and thereby expanding the chest and abdomen. When the negative pressure was released, the elastic recoil of the chest wall and the lungs led to expiration before the next cycle of negative pressure was generated. This treatment was widely used but led to problems when bulbar function was compromised, since it did not protect the airway. The importance of this was demonstrated in the massive 1952 Copenhagen poliomyelitis epidemic. Many patients were intubated and then ventilated by hand by medical students because of a lack of mechanical ventilators. The survival rate was greater than in those treated in iron lungs. ${ }^{4}$ This important observation led to the widespread use of positive pressure invasive ventilation for ventilatory failure and to the development of modern intensive care units.

It was soon recognised, however, that survival in patients with COPD was poor if they were invasively ventilated during an exacerbation, and a generation of physicians and anaesthetists came to believe that respiratory support was usually inappropriate for these patients. Ventilatory support for hypercapnic respiratory failure from the 1950s until the mid 1980s remained largely confined to patients with neuromuscular and skeletal disorders. Compact variants of the tank ventilators such as jacket and cuirass ventilators were developed for long-term use, and they prolonged the life expectancy, normalised blood gases and improved the quality of life. ${ }^{5}$ Other effective appliances were developed, including those that generated a positive expiratory pressure around the chest or abdomen, high-frequency chest wall oscillators, rocking beds which used gravitational force to compensate for a paralysed diaphragm, and phrenic nerve stimulators to make up for a defective medullary respiratory drive or damage to the respiratory pathways in the spinal cord. ${ }^{6}$ However, none of these treatments was widely applied, probably because of their cost, the difficulty in selecting appropriate patients and the considerable expertise required to make them work.

Even before the identification of rapid eye movement (REM) sleep in 1953 it was recognised that mechanical ventilatory support was effective if it was used only at night. Sleep was thought to be a vulnerable period for respiration during which voluntary control of breathing was absent and respiration relied on reflex pathways. Ideas about respiratory control during sleep and wakefulness have developed considerably since then, ${ }^{7}$ but ventilatory support at night usually remains sufficient to maintain normal arterial blood gases and to relieve symptoms of respiratory failure throughout the day.

The breakthrough in the mid 1980s was the development of comfortable and effective nasal and oronasal masks, with the confidence to apply positive pressure ventilation to the upper airway rather than through a tracheostomy. ${ }^{8}$ This relied on bulbar function to protect the tracheobronchial tree from aspiration. The ventilators for non-invasive positive pressure ventilation became simpler, smaller, had better triggering systems and were at least as reliable as the ventilators previously used for negative pressure ventilation. Mask ventilation was much more readily applied and more convenient for patients to use in the long term in their homes.

The evidence for the value of noninvasive positive pressure ventilation has unfortunately come mainly from case reports and retrospective series rather than randomised controlled trials. The studies have nevertheless shown an improvement in life expectancy, quality of life and blood gases, both during the day and at night in neuromuscular and skeletal disorders. ${ }^{9}{ }^{10}$ The situation is not so clear for COPD. A recent meta-analysis $^{11}$ of non-invasive ventilation in COPD has shown promising but not decisive results, although they are certainly better than with long-term invasive ventilation. ${ }^{12}$ In a cash-limited National Health Service where evidence is increasingly required to justify expenditure on treatments, it is particularly unfortunate that the evidence for the effectiveness of noninvasive ventilation in hypercapnic respiratory failure is so limited.

The use of non-invasive ventilation is spreading to patient groups with only mild respiratory failure or those who are at high risk of developing it. These include patients with Duchenne's muscular dystrophy, ${ }^{13}$ motor neurone disease and those with previous poliomyelitis in whom it is important to avoid excessive respiratory muscle activity because of the risk of developing the post poliomyelitis syndrome. Early intervention in some of these groups may well be effective, but the lessons from the recent past of not carrying out randomised controlled trials need to be learnt. Non-invasive ventilation is also becoming a standard technique in critical care units, both to avoid intubation and to assist in the weaning process. ${ }^{14}$ It is now widely used for acute exacerbations of $\mathrm{COPD}^{15}$ and in specialist units to wean these patients from invasive ventilation. ${ }^{16}$ There is little doubt that non-invasive ventilation will soon also become standard practice in treating the obesity hypoventilation syndrome as the obesity epidemic spreads through urbanised societies.
Despite these wide applications of ventilatory support for hypercapnic respiratory failure, the funding and resources-particularly of skilled medical and non-medical staff-are limited. While the provision of ventilatory support for acute respiratory failure should be provided in all acute district general hospitals, it is often difficult to provide continuously available high quality care. The issue of how much centralisation is best for long-term care also needs to be addressed. When the British Thoracic Society was established in 1982 virtually all non-invasive ventilation was provided in three units: the South Western Hospital in London (later transferred to St Thomas' Hospital) under the supervision of Geoffrey Spencer, Newmarket General Hospital (now at Papworth Hospital) under myself, and the Brompton Hospital under Margaret Branthwaite. Since then the provision of care has become much more widespread, although these three large units continue to care for many patients. Evidence regarding the value for money, quality of care and accessibility are issues that commissioners of health care are increasingly concerned about and which will determine the future pattern of provision of respiratory support in the UK.

The benefits from non-invasive ventilation show how the commonly held widespread negative attitudes about the outlook in respiratory failure need to be re-fashioned. New techniques will need to be assessed and the available ones better evaluated so that they can be targeted more precisely to those patients who are likely to benefit and so that home ventilation can be successfully integrated into the lives of both patients and carers.

Thorax 2007;62:1024-1026.

doi: $10.1136 /$ thx. 2007.090985

Correspondence to: Dr John Shneerson, Respiratory Support and Sleep Centre, Papworth Hospital, Cambridge CB23 8RE, UK; john. shneerson@papworth.nhs.uk

Competing interests: None.

\section{REFERENCES}

1 Shneerson JM. Respiratory failure in tuberculosis: a modern perspective. Clin Med 2004;4:72-6.

2 Campbell EJM. Respiratory failure. BM 1965;1:1451-60.

3 Drinker P, McKhann CF. The use of a new apparatus for the prolonged administration of artificial respiration. I. A fatal case of poliomyelitis. J Am Med Assoc 1929;92:1658-60.

4 Lassen HCA, ed. Management of life-threatening poliomyelitis, Copenhagen 1952-1956, with a survey of autopsy findings in 115 cases. Edinburgh and London: E and S Livingstone, 1956

5 Kinnear W, Hockley S, Harvey J, et al. The effects of one year of nocturnal cuirass-assisted ventilation in chest wall disease. Eur Respir J 1988;1:204-8.

6 Shneerson J. Disorders of ventilation. Oxford: Blackwell Scientific Publications, 1988. 
7 Shneerson JM. Respiratory failure during sleep in restrictive disorders. Int J Respir Care 2005; 1:17-21.

8 Kerby GR, Mayer LS, Pingleton SK. Nocturnal positive pressure ventilation via nasal mask. Am Rev Respir Dis 1987; 135:738-40.

9 Leger P, Bedicam JM, Cornette A, et al. Nasal intermittent positive pressure ventilation. Long-term follow-up with severe chronic respiratory insufficiency. Chest 1994;105:100-5.

10 Simonds AK, Elliott MW. Outcome of domiciliary nasal intermittent positive pressure ventilation in restrictive and obstructive disorders. Thorax 1995;50:604-9

11 Kolodziej MA, Jensen L, Rowe B, et al. Systematic review of noninvasive positive pressure ventilation in severe stable COPD. Eur Respir J 2007;30:293-306.

12 Robert D, Gerard M, Leger P, et al. La ventilation mecanique à domicile definitive par tracheotomie de I'insuffisant respiratoire chronique. Rev Fr Mal Respir 1983;1 1:923-36.

13 Raphael J-C, Chevret S, Chastang C, et al Randomised trial of preventive nasal ventilation in
Duchenne muscular dystrophy. Lancet 1994;343:1600-4

14 Garpestad E, Brennan J, Hill NS. Noninvasive ventilation for critical care. Chest 2007; 132:71 1-20.

15 British Thoracic Society. Guidelines: Non-invasive ventilation in acute respiratory failure. Thorax 2002;56:192-211.

16 Quinnell TG, Pilsworth S, Shneerson JM, et al Prolonged invasive ventilation following acute ventilatory failure in COPD: weaning results, survival and the role of noninvasive ventilation. Chest 2006;129:133-9.

\section{Chronic obstructive pulmonary disease past, present and future}

\section{P M A Calverley, J A Wedzicha}

\section{Progress in the understanding of COPD}

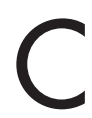
hronic obstructive pulmonary disease (COPD) is a topic of clinical concern and active research that is strongly represented at the meetings of the British Thoracic Society and in Thorax. This has not always been the case and, for rather different reasons, this disease may not be as important in future decades because the impact of COPD—at least in the developing world-should finally diminish over the next 20 years. This reflects the demographics of smoking cessation and the availability of more effective treatments to reduce the impact of this condition. The problems with COPD are not as current as is often stated. Necropsy data from the late 18th century identified the pathological features of emphysema while the clinical associations of this condition were described by René Laennec in his treaties on diseases of the chest in 1819. However, the slow pace of the illness and its lack of dramatic physical signs delayed our appreciation of its importance.

In the 20th century three partly related developments changed this. The first was the impact of warfare, specifically the use of toxic gases in World War I which stimulated research on pulmonary gas exchange. In World War II the need to fly at high altitudes without the pilots blacking out led to a renaissance of respiratory physiology which had its full impact when the relevant research was declassified at the end of hostilities. The ability to make objective measurements has transformed our understanding of COPD and coincided with a time when clinicians became aware of its importance, partly because of the accelerated death rate from respiratory causes including "bronchitis" after the 1952 London smog which linked impaired air quality very clearly with respiratory death. Additionally, Sir Richard Doll began his studies into the causes of death from lung cancer which led to the unequivocal relationship between tobacco smoking and this disease. As his studies on death in British doctors subsequently showed, the increase in deaths from lung cancer with rising tobacco consumption was paralleled by a similar increase in deaths due to chronic bronchitis and emphysema. ${ }^{1}$

Clinical terminology has proved to be a consistent barrier to progress in this field, and it was not until the late 1960s that it became clear that "British bronchitis" was clinically the same disease as "American emphysema". Compelling scientific data on the overlap of pathology between these clinically defined conditions was provided by the work of Hogg et $a l^{2}$ who established a clear association between pathological changes and abnormalities in pulmonary function, specifically airflow obstruction. A major step forward in our understanding of COPD came from the longitudinal study of Fletcher et al in Whitehall postal workers in whom symptoms and lung function were recorded over an 8-year period. ${ }^{3}$ Their initial hypothesis was that patients with symptoms of chronic bronchitis would show more deterioration. This did not prove to be the case and, instead, the initial level of lung function was a stronger predictor of their subsequent performance. As a result of these careful pathological and clinical observations, the role of airflow obstruction became central to the diagnosis and understanding of COPD and the preferred method of detecting this became spirometry. However, argument has continued over what to call this obstructive disease with a range of confusing synonyms being developed. Although COPD has eventually become the most widely used name, many clinicians still revert to chronic obstructive airways disease, chronic obstructive lung disease, chronic airflow limitation and even (although mainly in The Netherlands) chronic non-specific lung disease.

By the late 1970s the physiological basis of chronic airflow obstruction in terms of abnormal lung mechanics, gas exchange and the pulmonary circulation, at rest and during exercise, had largely been established. Unfortunately, much of the data was restricted to interested academics and did not diffuse into clinical practice until nearly 25 years later. Physiological studies did slowly begin to influence the way patients were managed. The development of controlled oxygen therapy was based on knowledge of oxygen delivery to the tissues and the risk of suppressing hypoxic ventilatory responses and of potentially modifying lung ventilation-perfusion relationships when patients were exposed to high concentrations of oxygen. This is a lesson which still has to be learnt in many emergency departments in the UK. The next step was to test the benefits of correcting persistent hypoxaemia in patients with more severe COPD where this was known to be a predictive factor for increased mortality. The resulting MRC and NIH oxygen trial provided the justification for domiciliary oxygen, not just in COPD but in many other conditions associated with chronic hypoxaemia. ${ }^{4}$ During the 1980 s we failed to build on these pioneering studies, but in the last 15 years we have seen important large-scale clinical trials addressing the effect of smoking cessation in COPD, ${ }^{6}$ the role of long-acting inhaled bronchodilators and corticosteroids in changing clinical outcome potentially affecting 